Ann. Sci. forest., 1973, 30 (2), 157-173.

\title{
ESSAIS PRÉLIMINAIRES SUR LA SÉLECTION DE CLONES BOUTURABLES DE DOUGLAS (PSEUDOTSUGA MENZIESII FRANCO)
}

\author{
D. CORNU \\ Station d'Amélioration des Arbres forestiers, \\ Centre national de Recherches forestières, I.N.R.A., \\ Champenoux 54370 Einville
}

\begin{abstract}
RÉSUMÉ
En vue de l'obtention de clones de Douglas à bonne aptitude à la reproduction par bouturage, trois expérimentations ont été conduites en 1970 et 1972 à partir de sujets sélectionnés. Les meilleurs résultats ont été obtenus à l'aide à la fois de boutures latérales et par un poudrage de la base avec un mélange à $0,5 \%$ d'A.I.B.

Les résultats moyens sont de $41 \%$ pour les 27 clones essayés en 1970 et de $63,5 \%$ pour 14 clones choisis parmi ceux-ci et réessayés en 1972. A cette date, les 14 clones étaient âgés de 10 ans. Parallèlement, on a comparé l'aptitude de 49 clones âgés de 60 ans; les résultats moyens sont nettement inférieurs : $18 \%$. On note une bonne concordance des enracinements observés en 1970 et 1972 sur les 14 clones communs aux deux expériences. Dans toutes les expérimentations, la variabilité individuelle entre clones est très grande. Parmi les populations étudiées, pour un traitement donné, environ $20 \%$ des clones peuvent être jugés supérieurs et sélectionnables pour leur aptitude au bouturage. Les résultats enregistrés laissent ressortir que les épreuves devraient être répétées plusieurs années et probablement mettre en jeu plusieurs concentrations auxiniques. Un maximum de clones pourra alors être sélectionné avec certitude pour leur bonne aptitude à la propagation par bouturage.
\end{abstract}

\section{INTRODUCTION}

Le Douglas (Pseudotsuga menziesii Franco) est à l'heure actuelle la première essence de reboisements en France. Il s'adapte à un assez large éventail de conditions écologiques, présente une croissance juvénile rapide et produit tant quantitativement que qualitativement un bois intéressant, d'où l'intérêt des études effectuées. La Station d'Amélioration des Arbres forestiers a entrepris depuis plusieurs années un programme d'amélioration du Douglas. L'infrastructure de travail est constituée par diverses plantations comparatives de provenances ou de descendances Nord-Américaines et Françaises ainsi que par des parcs à clones obtenus par greffage d'arbres sélectionnés dans les peuplements français. 
Nos études sur le bouturage du Douglas s'intègrent à deux niveaux dans ce programme :

1. Réalisation de vergers à graines de clones à partir de boutures. Le greffage, technique habituelle pour la constitution de ces vergers, bute sur le problème de l'incompatibilité portegreffe / greffon. Incompatibilité d'autant plus préjudiciable aux expériences, qu'elle peut intervenir tardivement, après la mise en place définitive des sujets greffés.

2. Création de variétés multiclonales à partir de génotypes supérieurs sélectionnés dans les plantations comparatives de provenances ou de descendances, et peut-être après rajeunissement et confirmation, de clones sélectionnés en forêt.

Le bouturage du Douglas a fait l'objet d'un certain nombre de travaux. (Bouvarel et Turpin, 1955 (non publié), Roberts 1969, Heinrich 1969, Brix et Barker 1969 et 1971, Heaman et Owens 1972). Ceux-ci montrent que : le Douglas s'enracine relativement bien à l'état jeune mais très difficilement à l'état âgé; pour obtenir un bon enracinement, un traitement à l'aide de substances de croissance de type auxinique est généralement nécessaire; la réponse maximum est généralement obtenue durant la période de repos hivernal.

En nous appuyant sur ces résultats, nos travaux ont eu pour but de tester l'aptitude au bouturage d'un certain nombre de sujets sélectionnés en 1970 et 1972.

\section{I. - MÉTHODE}

\section{1. - Le matériel végétal étudié}

\subsection{Origine.}

Nous avons travaillé sur deux types de sujets issus soit de plantation comparative soit de peuplements adultes.

\subsection{Plantation comparative.}

En 1970, nous avons sélectionné 27 sujets dans une plantation de 21 provenances à Épinal. Les sujets avaient alors 8 ans dont 5 de plantation. La sélection a été effectuée sur 3 critères importants, les deux premiers analysés à partir des fiches des mesures de terrain, le troisième jugé directement sur place lors de la récolte :

— tardiveté du débourrement et absence de dégâts de gelées tardives;

- croissance;

— port général, les sujets tordus, fourchus et surtout fastigiés ont été systématiquement éliminés.

\subsection{Peuplements adultes.}

Nous avons utilisé les sélections effectuées par le responsable de l'amélioration de cette espèce dans des peuplements du Beaujolais sur des critères de tardiveté du débourrement et de densité du bois. Les sujets ont en moyenne 50 à 60 ans.

\subsection{Prélèvement et préparation des boutures (figure 1).}

Celles-ci ont été prélevées au cours des mois de février et mars en 1970 et 1972. Sur les sujets de plantation comparative, les boutures sont issues du troisième verticille à partir du sommet. Chez les sujets adultes, ce sont des pousses vigoureuses de la zone supérieure de l'arbre.

Après prélèvement, les rameaux sont stockés en sac de polyéthylène en chambre froide à $+4{ }^{\circ} \mathrm{C}$ jusqu'à la mise en place des boutures.

D’une manière générale, nos boutures ont une longueur de $15 \mathrm{~cm}$ et sont constituées exclusivement à partir des pousses formées l'année précédente. Avant le traitement et la mise en place, les aiguilles de la base sont ôtées sur environ 3 à $4 \mathrm{~cm}$. 

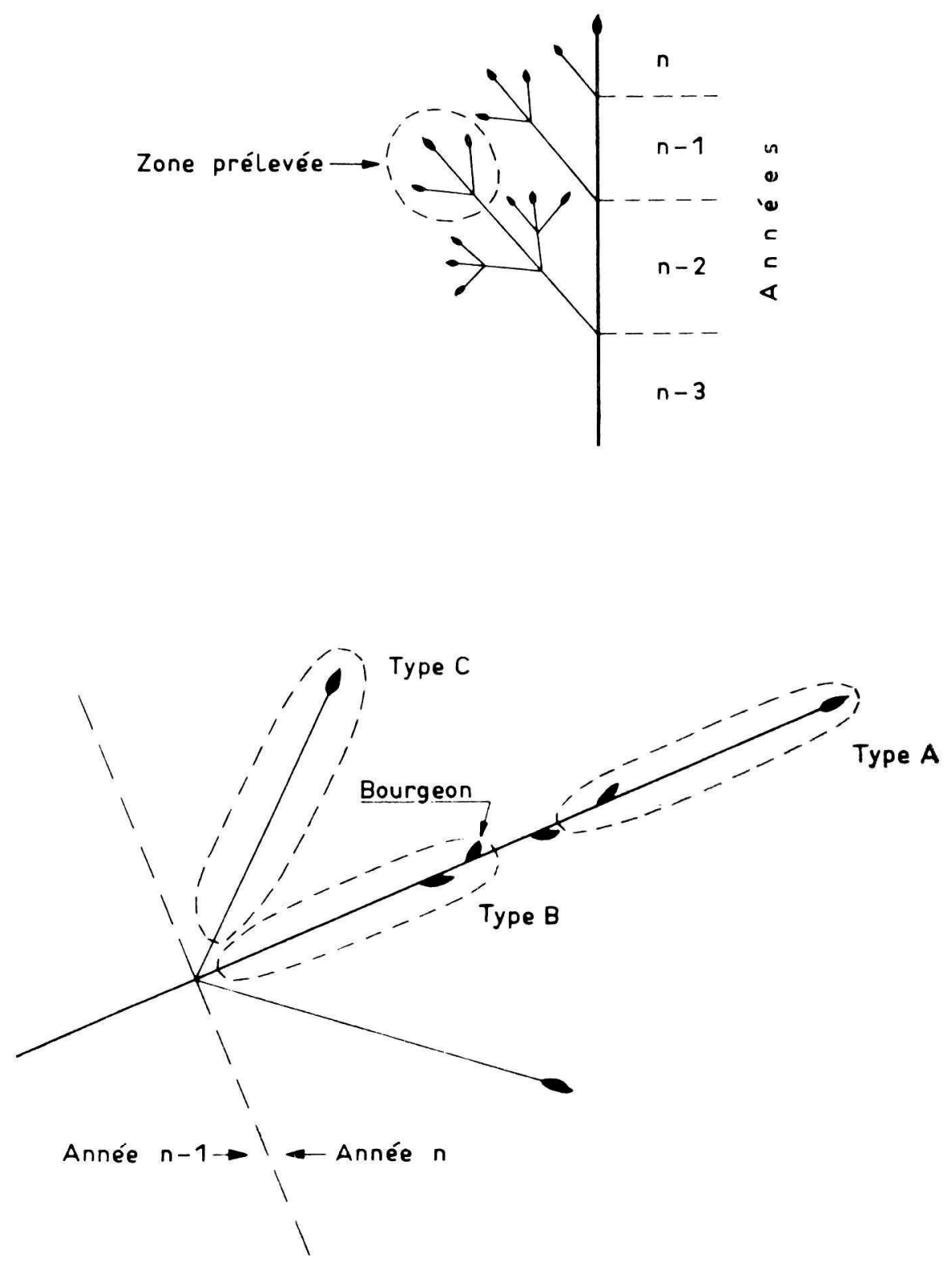

FIG. 1. - Zone de prélèvement des boutures (Épinal) et type des boutures utilisées. Année de prélèvement $: \mathrm{n}+\mathrm{l}$.

FIG. 1. - Sampling area (Epinal) and types of cuttings used. Sampling year: $\mathbf{n}+1$. 


\section{2. - Le milieu de bouturage}

Les boutures sont installées sous une serre abri tunnel recouverte de chlorure de polyvinyl (figure 2). Un ombrage à $50 \%$ est adjoint à cette serre. Cet abri est sommairement climatisé. En 1970 seulement à l'aide d'un chauffage à air pulsé qui nous permettait de maintenir l'enceinte hors-gel. En période hivernale normale, nous pouvons maintenir une température de $+5^{\circ}$ à $+10{ }^{\circ} \mathrm{C}$ la nuit, et $+15^{\circ}$ à $+25^{\circ} \mathrm{C}$ le jour. Lors des journées ensoleillées de printemps, la température fortement tributaire des conditions externes, était souvent assez élevée : $30{ }^{\circ} \mathrm{C}$.

En 1972, nous avons installé un système de ventilation qui nous permet d'écrêter ces hausses de température à $25^{\circ} \mathrm{C}$ environ.

Le substrat était constitué de sable de rivière de granulométrie $0-5 \mathrm{~mm}$ (dont $50 \%$ de $0-2$ ). Après nos essais de 1970, n ous avons procédé en 1972 à l'élimination de la fraction sableuse de granulométrie 0-2 pour obtenir un meilleur drainage de l'eau apportée.

L'irrigation est assurée par des diffuseurs nébulisateurs sur rampe. La fréquence des arrosages est réglée à l'aide d'une feuille électronique (ou feuille artificielle) afin que les boutures soient toujours humides.

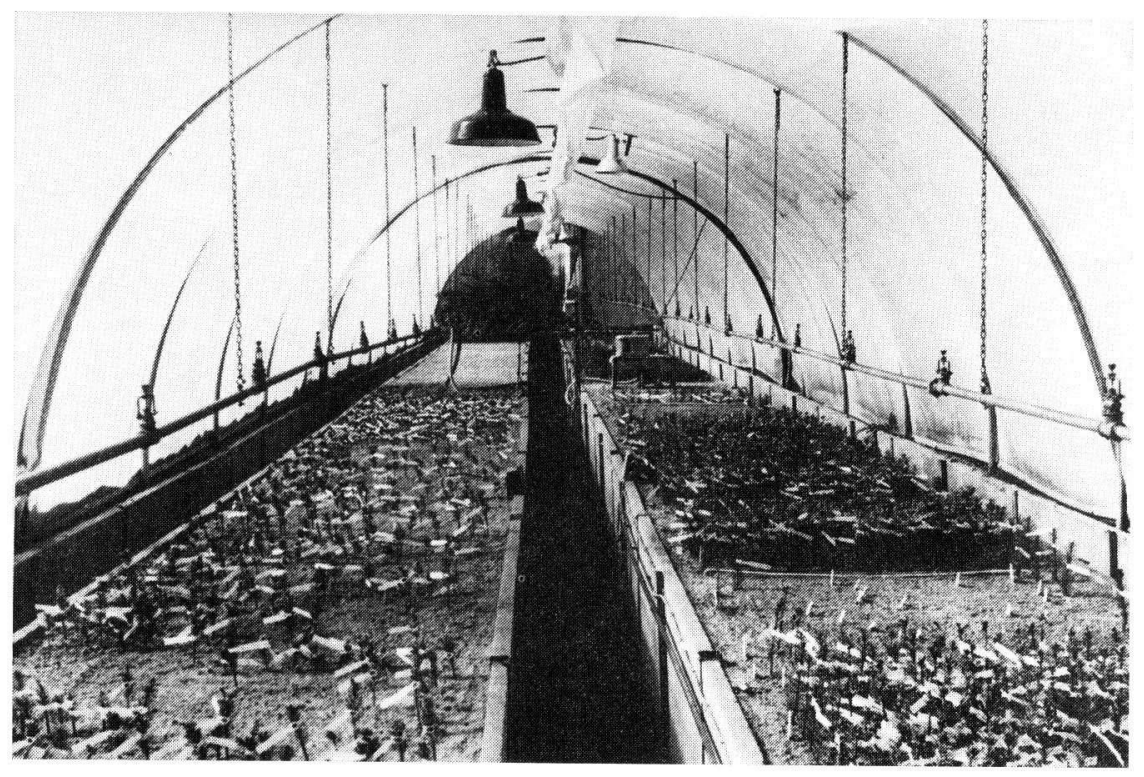

FIG. 2. - Installation utilisée pour les expérimentations de bouturage (boutures restantes après lecture à 6 mois).

FIG. 2. - Device used for the experimentation (cuttings left after result uptake 6 months).

\section{3. - Traitement et mise en place}

Nous avons distingué 3 types de boutures A - B - C, suivant leur position sur le rameau (figure 1) :

- A : pousse terminale du rameau. Elle est en général très vigoureuse sur les sujets jeunes;

- B : partie basale, avec au moins 1 bourgeon, de la pousse précédente lorsque celle-ci était suffisamment grande (supérieure à $30 \mathrm{~cm}$ );

- C : pousses latérales à la base de la précédente.

Suivant les quantités disponibles pour chaque clone, les boutures sont réparties en 2 ou 3 répétitions de 5 ou 10 pour chaque traitement. 
Les boutures ont été soumises à l'action d'une substance auxinique, l'A.I.B. (') apporté par poudrage. Dans chaque expérience existe un traitement témoin par poudrage avec du talc pur. Nous avons utilisé les concentrations en matière active suivantes :

1970 : boutures d’Épinal $1 \%$ d'A.I.B.;

1972 : boutures d'Épinal 0,5\%-1\%-2\% d'A.I.B.;

1972 : boutures du Beaujolais 0,5\% d'A.I.B.

Les expérimentations ont porté pour la plantation comparative d'Épinal sur 27 clones (1 920 boutures) en 1970 et sur 14 clones (500 boutures), en 1972, choisis parmi les précédents pour leur très bonne moyenne ou leur très mauvaise aptitude au bouturage. Nous avons testé 49 clones (1 700 boutures) récoltés dans le Beaujolais en 1972. Les boutures ont été installées aux mois de mars 1970 et mars et avril 1972 suivant les dates de récoltes (environ 1 mois après celles-ci).

\section{4. - Contrôle des expériences}

Après leur mise en place, les boutures ont subi à intervalle plus ou moins régulier ( 3 semaines environ) un traitement antifongique. Les boutures mortes sont ôtées afin de prévenir toute extension de pourriture.

Les lectures ont été effectuées en 1970:8 mois et en $1972: 6$ et 8 mois, après la mise en place. Les boutures sont alors classées en 3 catégories :

— boutures mortes : perte des aiguilles à la manipulation, partie basale plus ou moins putréfiée sans zone cicatricielle;

— boutures vivantes : toute bouture saine avec un cal plus ou moins important;

- boutures enracinées : toute bouture avec au moins une racine visible.

Après lecture, les boutures enracinées sont repiquées en godet- « Fertil »10 $\times 18$ dans un mélange sable-terre de bruyère 1 - 1. Repérées individuellement, ces boutures sont conservées afin d'étudier ultérieurement leur développement tant au point de vue croissance aérienne que souterraine.

\section{II. - RÉSULTATS}

Nous pouvons classer nos résultats en deux catégories, celle relative aux données générales, et celle relative à la variabilité individuelle.

\section{1. - Données générales}

\subsection{Action d'un traitement auxinique.}

A la lecture des tableaux 1 et 4 , nous observons une très forte influence du traitement auxinique sur l'enracinement. Le gain obtenu est très net et très important. C'est un résultat qui confirme ceux obtenus par d'autres chercheurs, Bouvarel et Turpin (1955), Heinrich (1969) et nous-mêmes.

Nous l'expliciterons plus loin, mais nous pouvons déjà remarquer que dans les conditions de 1972 (tableau 4), une formulation de 0,5\% d'A.I.B. s'est révélée donner la meilleure réponse au niveau de l'ensemble des clones.

Contrairement aux résultats que nous avions obtenus précédemment au Québec (mortalité de $17 \pm 2 \%$ pour traitement et témoin), nous pouvons observer une très grande action du

(1). A.I.B. = acide indol butyrique. 


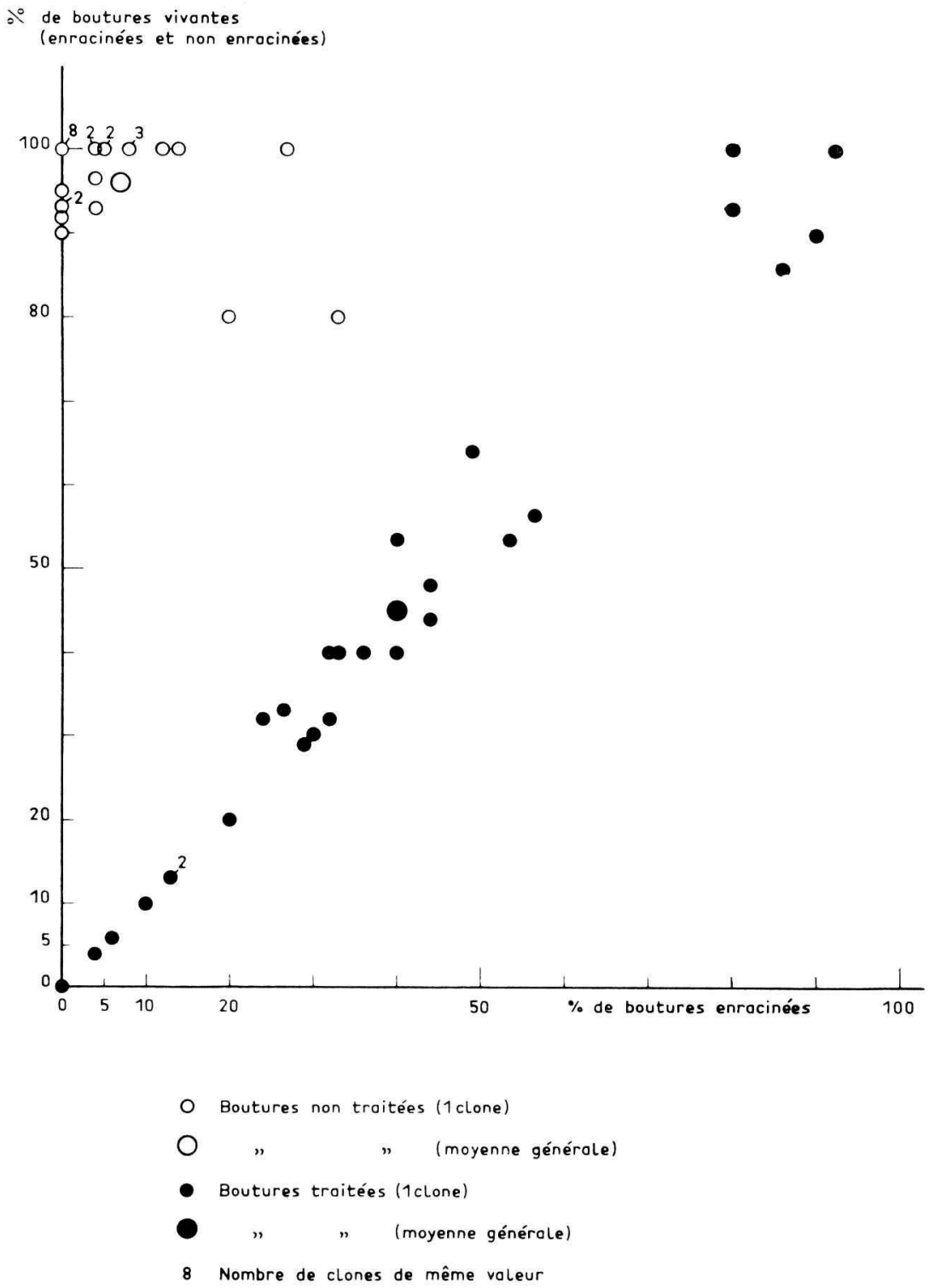

FIG. 3. - Action d'un traitement auxinique (A.I.B. $1 \%$ ) sur la survie et l'enracinement (Épinal - $1970-27$ clones).

FIG. 3. - Effects of $1 \%$ I.B.A. treatment on survival and rooting (Epinal - $1970-27$ clones).

traitement auxinique sur la mortalité. Très faible pour les boutures témoins, elle touche par contre pratiquement toutes les boutures traitées non enracinées (tableaux 1 et 3 , figure 3 ). Nous pouvons penser que cette mortalité peut être liée à l'action de l'auxine sur la prolifération des tissus cicatriciels. En effet, nous avons observé que les boutures mortes présentaient 


\section{TABLEAU 1}

Action d'un traitement auxinique sur l'enracinement et la mortalité Pourcentage de boutures (analyse sur les boutures de type $C$ ) Traitement: A.I.B. $1 \%$

Effects of I.B.A. treatment on death and rooting percentages.

\begin{tabular}{|c|c|c|c|c|c|c|}
\hline & \multicolumn{2}{|c|}{ Enracinées } & \multicolumn{2}{|c|}{ Mortes } & \multicolumn{2}{|c|}{$\begin{array}{c}\text { Vivantes } \\
\text { non enracinées }\end{array}$} \\
\hline & Témoin & A.I.B. & Témoin & A.I.B. & Témoin & A.I.B. \\
\hline $\begin{array}{l}1970 \text { Épinal : } 27 \text { clones } 20 \text { bou- } \\
\text { tures par clone et par traite- } \\
\text { ment ( } 540 \text { boutures) }\end{array}$ & 7 & 41 & 3 & 55 & 90 & 4 \\
\hline $\begin{array}{l}1972 \text { Épinal : } 14 \text { clones } 10 \text { bou- } \\
\text { tures par clone et par traite- } \\
\text { ment ( } 140 \text { boutures) }\end{array}$ & 1,5 & 63,5 & 14 & 34,5 & 84,5 & 2 \\
\hline
\end{tabular}
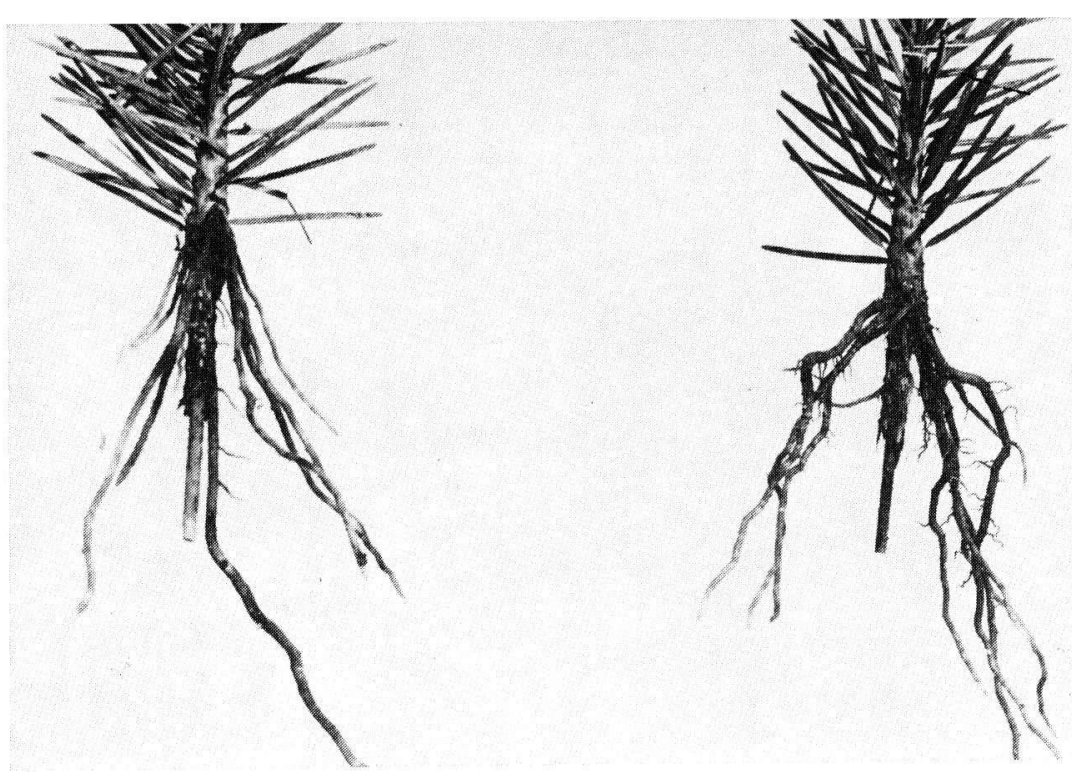

FIG. 4. - Boutures enracinées dont la partie enfouie est morte par excès d'humidité (Épinal - 1970-A.I.B. $1 \%$ )

FIG. 4. - Rooted cuttings on which the buried part has died by excess of moisture (Epinal-1970-I.B.A. $1 \%$ ). 

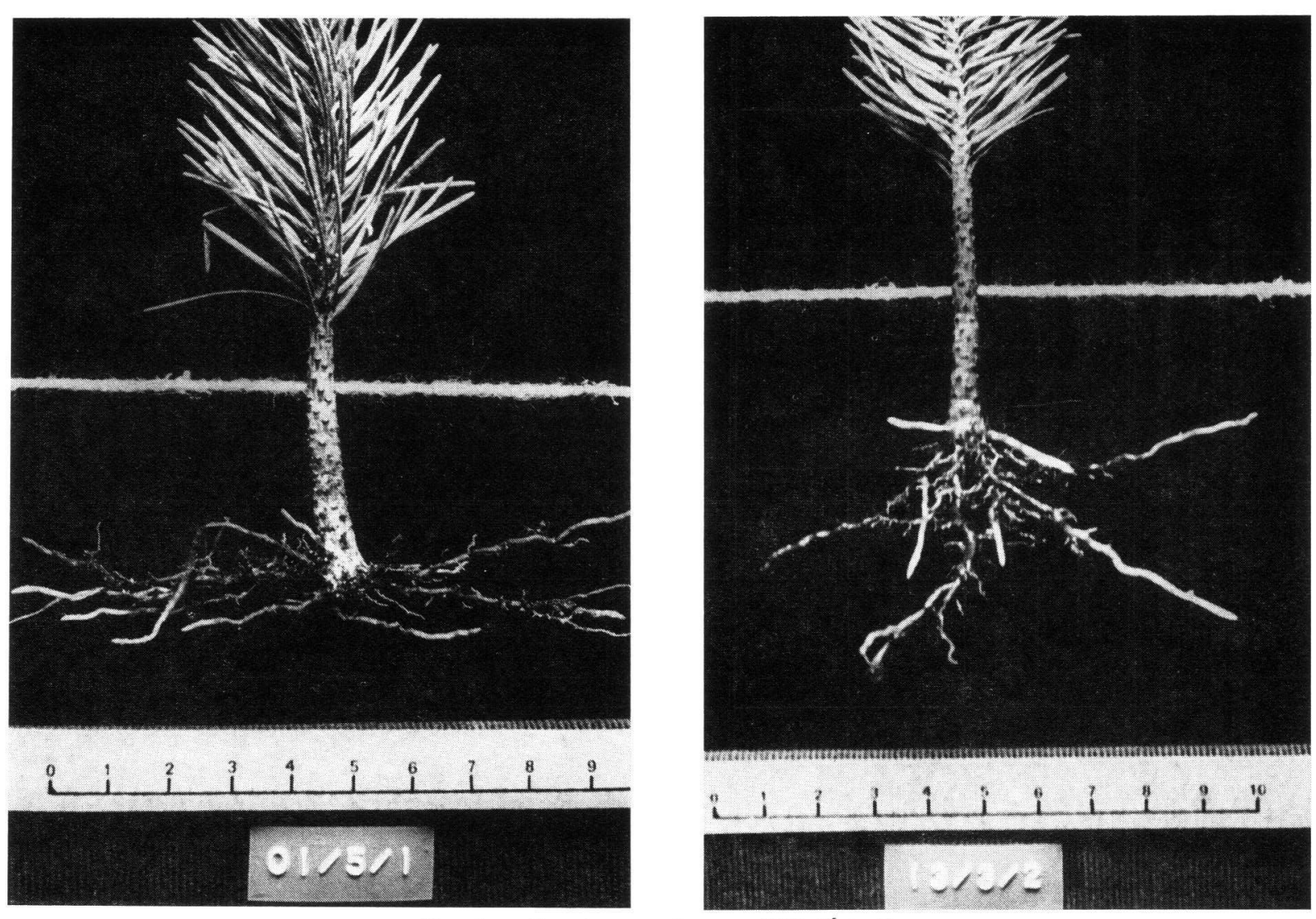

$J$
$\delta$
$\frac{\pi}{\pi}$
ż

FIG. 5. - Enracinement obtenu en 1972 (Epinal)

clone 01 - A.I.B. 0.5\%. Clone 13 - A.I.B. $1 \%$

FIG. 5. - Rooting obtained in 1772.

clone 01 - I.B.A. 0,5\%. Clone $13-$ I.B.A. $1 \%$; 
souvent un cal important putréfié ainsi qu'un éclatement de l'épiderme au niveau de la partie enfouie, alors que les boutures témoins vivantes avaient de petits cals durs. Les boutures ayant ces tissus friables importants ou ces blessures ont probablement été beaucoup plus sensibles aux conditions défavorables (en particulier excès d'humidité) qui ont parfois existé. Nous pouvons compléter cette remarque par des observations faites en 1970 selon lesquelles environ 1/4 des boutures enracinées présentaient une destruction plus ou moins importante de la partie enfouie (figure 4). Pour ces boutures, les processus de cicatrisation et de rhizogénèse ont pu dominer les processus de destruction.

Les résultats obtenus en 1972 sont dans l'ensemble meilleurs tant au point de vue pourcentage d'enracinement, que qualité de cet enracinement. Les boutures enracinées ont en moyenne un enracinement plus équilibré et plus puissant (figure 5). Nous attribuons ce phénomène à l'amélioration du milieu que nous avons effectuée, en particulier le drainage. Néanmoins, nous n'avons pas encore obtenu les conditions les plus favorables. En 1972, le taux d'irrigation a encore été trop fort, ce qui a entraîné une certaine destruction des nouvelles pousses.

\subsection{Influence du type de pousse utilisée.}

Chaque fois que cela a été possible (quantité suffisante de matériel), nous avons comparé les boutures de type $\mathrm{A}$ et $\mathrm{B}$ avec celles de type $\mathrm{C}$ communément utilisées. Les résultats sont résumés dans le tableau 2.

TABLEAU 2 Pourcentages d'enracinement de différents types de boutures. A. B. C. (fig. 1)
Épinal 1970 et 1972

Traitement: A.I.B. $1 \%$

Rooting percentage of different types of cuttings: A. B. C.

\begin{tabular}{|c|c|c|c|c|c|c|}
\hline \multirow{2}{*}{ Clones } & \multicolumn{2}{|c|}{ Type A } & \multicolumn{2}{|c|}{ Type B } & \multicolumn{2}{|c|}{ Type C } \\
\hline & 70 & 72 & 70 & 72 & 70 & 72 \\
\hline $\begin{array}{l}01 \\
02 \\
03 \\
09 \\
12 \\
13 \\
19 \\
23\end{array}$ & $\begin{array}{r}20 \\
11 \\
15 \\
0 \\
33 \\
8 \\
0 \\
0\end{array}$ & $\begin{array}{l}0 \\
0 \\
0 \\
0 \\
0 \\
0 \\
0 \\
0\end{array}$ & $\begin{array}{r}33 \\
20 \\
10 \\
0 \\
50 \\
0 \\
38 \\
20\end{array}$ & $\begin{array}{r}0 \\
0 \\
20 \\
0 \\
40 \\
20 \\
40 \\
0\end{array}$ & $\begin{array}{r}80 \\
87 \\
92 \\
57 \\
90 \\
30 \\
7 \\
4\end{array}$ & $\begin{array}{r}100 \\
60 \\
100 \\
100 \\
100 \\
70 \\
30 \\
20\end{array}$ \\
\hline Moyenne & 11 & 0 & 21 & 15 & 56 & 72 \\
\hline
\end{tabular}

Les boutures de type $C$ sont très nettement supérieures aux autres. De même, les boutures de type $\mathrm{B}$ prélevées à la base des pousses terminales vigoureuses sont légèrement supérieures aux extrémités de ces mêmes pousses. Ces résultats sont identiques dans les trois expériences que nous avons effectuées. 
ROBERTS (1969) trouve que des boutures terminales s'enracinent au moins aussi bien que des boutures latérales. Néanmoins, avant le débourrement, un traitement auxinique diminue très fortement l'enracinement de ces boutures. RoBERTs attribue à l'état d'évolution du bourgeon un rôle très important sur ce processus. Des différences notables existent entre les types de boutures que nous avons étudiés quant à la grosseur et probablement quant à l'état de développement des bourgeons. Elles pourraient expliquer les variations observées. Toutefois, une étude plus approfondie est nécessaire afin de définir les facteurs responsables de ces différences et surtout ceux responsables des très faibles pourcentages obtenus avec les boutures de type A.

\subsection{Stabilité de l'aptitude à l'enracinement.}

Les données sont résumées dans le tableau 3 et la figure 6 . Nous pouvons observer une très bonne stabilité de l'enracinement entre les campagnes de 1970 et 1972 . Toutes restrictions faites sur la faible quantité de couples (14) analysés, nous obtenons un coefficient de corrélation de 0,72 significatif au seuil de 0,01 .

TABLEAU 3

Pourcentages d'enracinement et de mortalité pour 14 clones d'Épinal Saisons 1970 et 1972

Traitement: A.I.B. $1 \% 20$ boutures par clone et par traitement en 1970 10 boutures par clone et par traitement en 1972

Rooting percentage and death rate of 14 clones of Epinal.

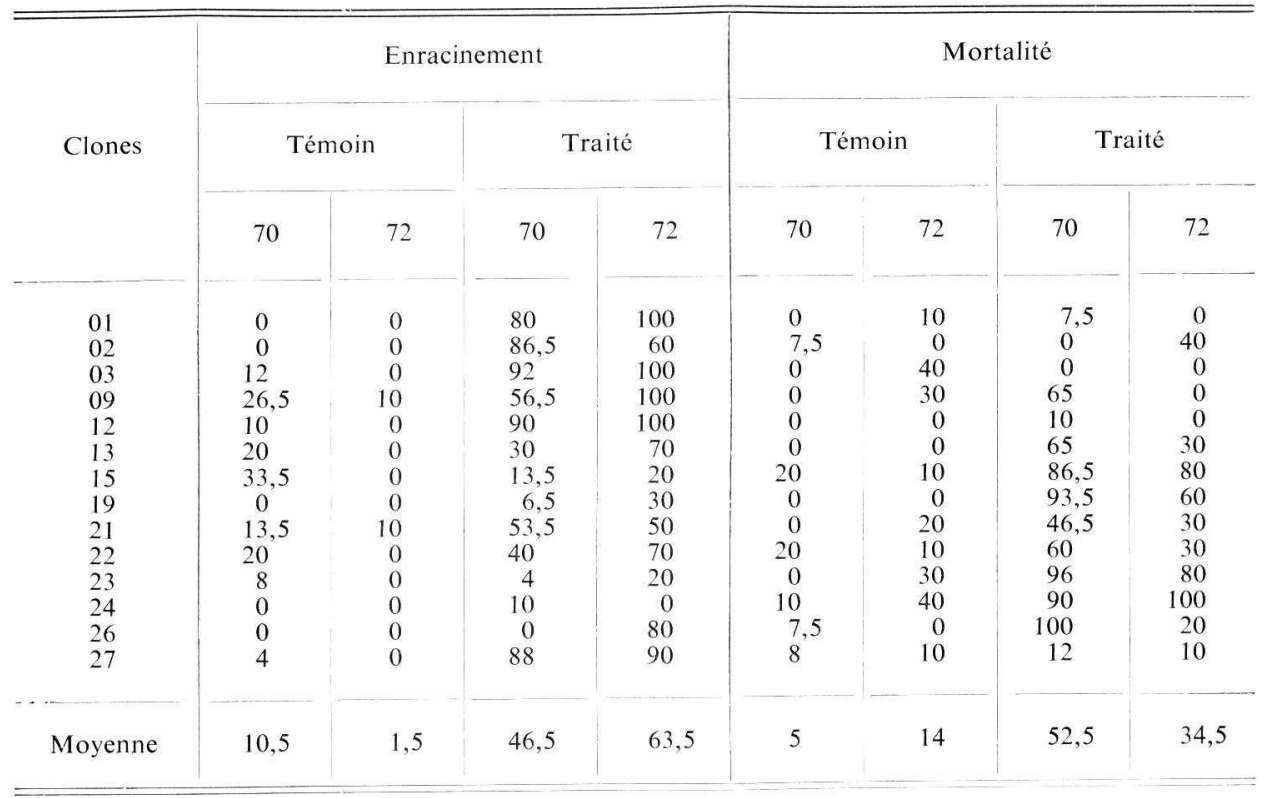

Si nous tenons compte de la meilleure réussite obtenue en moyenne en 1972, nous pouvons observer que seuls 3 clones $(02,09$ et 26$)$ sur les 14 présentent des résultats nettement discordants. 


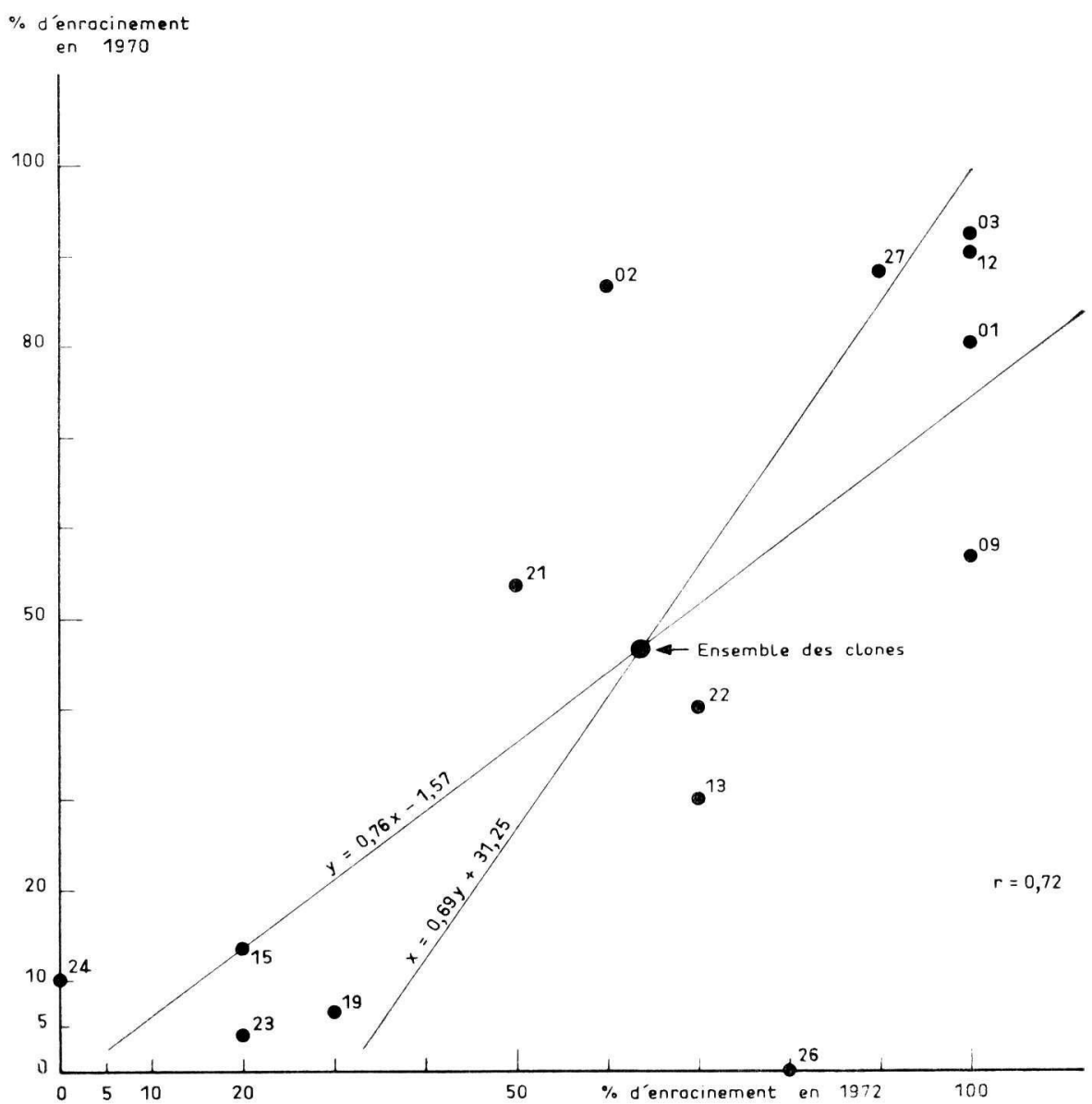

FIG. 6. - Comparaison de l'enracinement de 14 clones récoltés en 1970 et 1972 (Épinal - A.I.B. $1 \%$ ). FIG. 6. - Rooting comparison of 14 clones collected in 1970 and 1972 (Epinal - I.B.A. $1 \%$ ).

Plutôt qu'à une variation de l'aptitude à l'enracinement de ces clones entre 1970 et 1972, nous pouvons penser que ces discordances peuvent être la conséquence d'accidents, accidents multiples qui auraient pu intervenir aussi bien avant qu'après le prélèvement. Le clone 26 présente peut-être une sensibilité particulière à un excès d'humidité du milieu, situation ayant régné en 1970 .

Des 5 clones jugés en 1970 particulièrement réfractaires au bouturage, 4 confirment leur particularisme en 1972. De même, des cinq clones susceptibles d'être sélectionnés en 1970 pour leur très bonne aptitude, un seul présente une réponse moyenne en 1972.

Malgré l'observation précédente, juger un clone sur une seule expérimentation présente des risques (en l'occurence l'élimination du clone 26 en 1970). Nous pensons qu'un minimum de 3 tests serait nécessaire pour obtenir une bonne idée de l'aptitude réelle d'un clone au bouturage. 
En 1972, les clones âgés de 10 ans ont un taux d'enracinement moyen encore élevé. Par contre, ceux âgés de 60 ans présentent un taux très nettement inférieur. Ces résultats sont voisins de ceux obtenus par BRIX et BARKER (1971) qui trouvent pour des sujets de 5 ans un taux d'enracinement de $54 \%$ et de seulement $9 \%$ pour des sujets de plus de 60 ans. Dans les plantations comparatives, nous disposons d'un volume de mesures de plus en plus grand au fil des ans. Pouvoir attendre plusieurs années avant de procéder à un test d'enracinement, tout en conservant une bonne aptitude générale au bouturage, permet une meilleure connaissance des individus, donc une sélection plus rigoureuse. Néanmoins, tant que n'auront pas été définies les corrélations juvénile-adulte, les clones devront continuer à être suivis en plantation afin de connaître leurs performances.

\section{2. - Variabilité individuelle}

Comme nous le montrent le tableau 3 et la figure 7, la variabilité de l'enracinement est très importante (de 0 à $100 \%$ ). Bien que nous observions un certain tassement vers les taux d'enracinement très faibles ou nuls, cette variabilité est encore très fortement marquée pour les clones du Beaujolais, comme nous le montre l'analyse de variance suivante effectuée après transformation angulaire.

\begin{tabular}{|c|c|c|c|c|}
\hline $\begin{array}{l}\text { Source de } \\
\text { variation }\end{array}$ & $\begin{array}{l}\text { Somme des } \\
\text { carrés }\end{array}$ & d.d.I. & $\begin{array}{l}\text { Carrés } \\
\text { moyens }\end{array}$ & $\mathrm{F}$ \\
\hline $\begin{array}{l}\text { clones } \\
\text { blocs } \\
\text { erreurs } \\
\text { total }\end{array}$ & $\begin{array}{r}30077,15 \\
290,96 \\
14157,28 \\
44525,39\end{array}$ & $\begin{array}{r}48 \\
2 \\
96 \\
146\end{array}$ & $\begin{array}{l}626,61 \\
145,48 \\
147,47\end{array}$ & $4,25\left(^{(1)}\right.$ \\
\hline
\end{tabular}

(1) $\mathrm{P}<0,01$.

Dans les deux populations étudiées, nous pouvons observer qu'un petit nombre d'individus se détache et présente une aptitude au bouturage nettement supérieure à celle des autres. Nous avons peut-être pour ces sujets un particularisme génétique qui pourrait être relativement simple. Bouvarel et Turpin (1955) (non publié) avaient observé le même phénomène dans 3 expérimentations, portant chacune sur environ 50 clones.

Néanmoins, une étude plus complète à partir de sujets et de leurs descendants obtenus par pollinisation contrôlée serait nécessaire pour définir le déterminisme génétique de l'aptitude au bouturage.

Ces sujets que nous pourrions dénommer « supérieurs », sont les clones sélectionnables. Ils représentent $20 \%$ des populations aussi bien d'Épinal que du Beaujolais. Des taux de sélection voisins avaient été obtenus par Bouvarel et TurPIN (1955) et par HeINRICH (1969) ( $25 \%$ après 6 mois et $15 \%$ après 1 an d'expérimentation).

Nous avons travaillé sur un échantillon biaisé par la sélection (sous population de tardifs). Au cas où il existerait des corrélations génétiques défavorables pour les critères de sélection importants, en particulier la densité, il est évident que l'intensité de sélection serait beaucoup plus importante. 

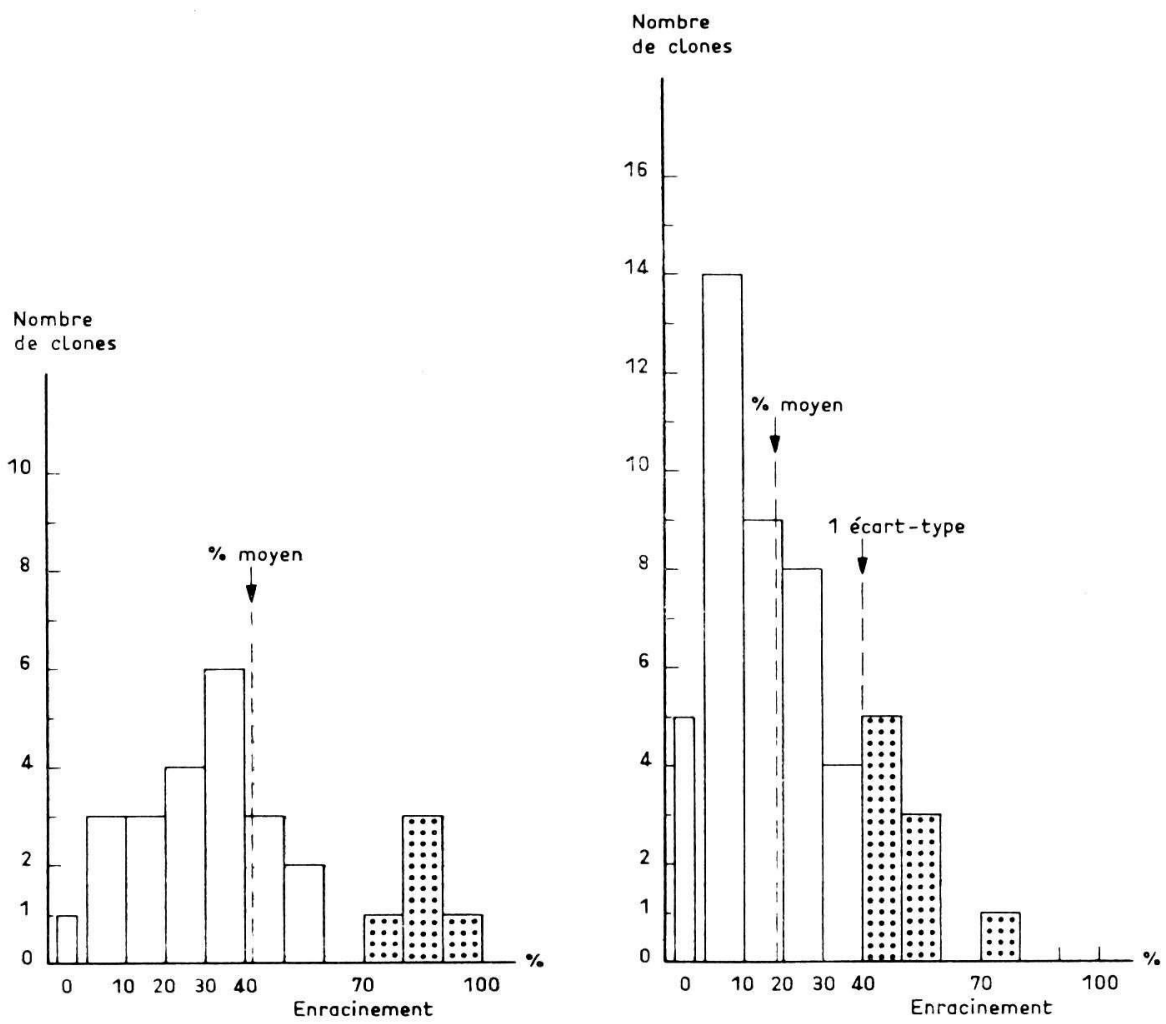

$::$ Clones sélectionnables pour ::: L'aptitude ou bouturage

FIG. 7. - Répartition des clones par classe selon leur enracinement.

FIG. 7. - Distribution of the clones according to their rooting.

A : Épinal 1970, 27 clones, âge 8 ans.

Traitement A.I.B. $1 \%$.

Pourcentage moyen d'enracinement : $42 \%$.

A : Epinal 1970, 27 clones, age : 8.

Treatment I.B.A. $1 \%$.

Average rooting percentage : $42 \%$.

B : Beaujolais 1972, 49 clones, âge moyen 60 ans. Traitement A.I.B. $0,5 \%$.

Pourcentage moyen d'enracinement : $18 \%$.

$B$ : Beaujolais 1972, 49 clones, mean age : 60. Treatment I. B. A. 0,5\%.

Average rooting percentage : $18 \%$. 
Nous n'avons utilisé dans nos essais qu'une seule substance de croissance, l'A.I.B. et un mode d'application par poudrage qui n'est guère quantitatif. Ces restrictions faites, nous pouvons observer que la variabilité clonale s'exprime aussi dans la réponse au traitement auxinique (tableau 4). Pour la majorité des clones, l'optimum de réponse est obtenu avec $0,5 \%$ d'A.I.B. Une augmentation de la concentration à $1 \%$ a un effet très variable suivant les clones. Pratiquement nulle pour 4 de ceux-ci, elle $\mathrm{a}$, par contre, une action très positive pour le clone 27 et très dépressive pour les clones 15 et 23 . Dans pratiquement tous les cas, une concentration de $2 \%$ a eu un effet dépressif. Elle a cependant permis au clone 24 de donner 2 boutures enracinées. HeINRICH (1969) note aussi une décroissance de l'enracinement avec des doses croissantes d'A.I.B., décroissance très nette pour $2 \%$ d'A.I.B. Les besoins en auxine exogène très variables, doivent révéler des différences physiologiques entre les clones au niveau des substances de croissance. Selon les conclusions de Roberts (1969) sur le rôle des bourgeons, un état variable de leur développement ou de leur activité entre les clones pourrait expliquer ces résultats.

TABLEAU 4

Réaction de 11 clones à différentes concentrations croissantes d'A.I.B.

Pourcentages d'enracinement

Épinal 1972. Boutures de type $C$.

Observations portant sur 10 boutures par clone

Effect of I.B.A. increasing concentrations on the rooting of 11 clones

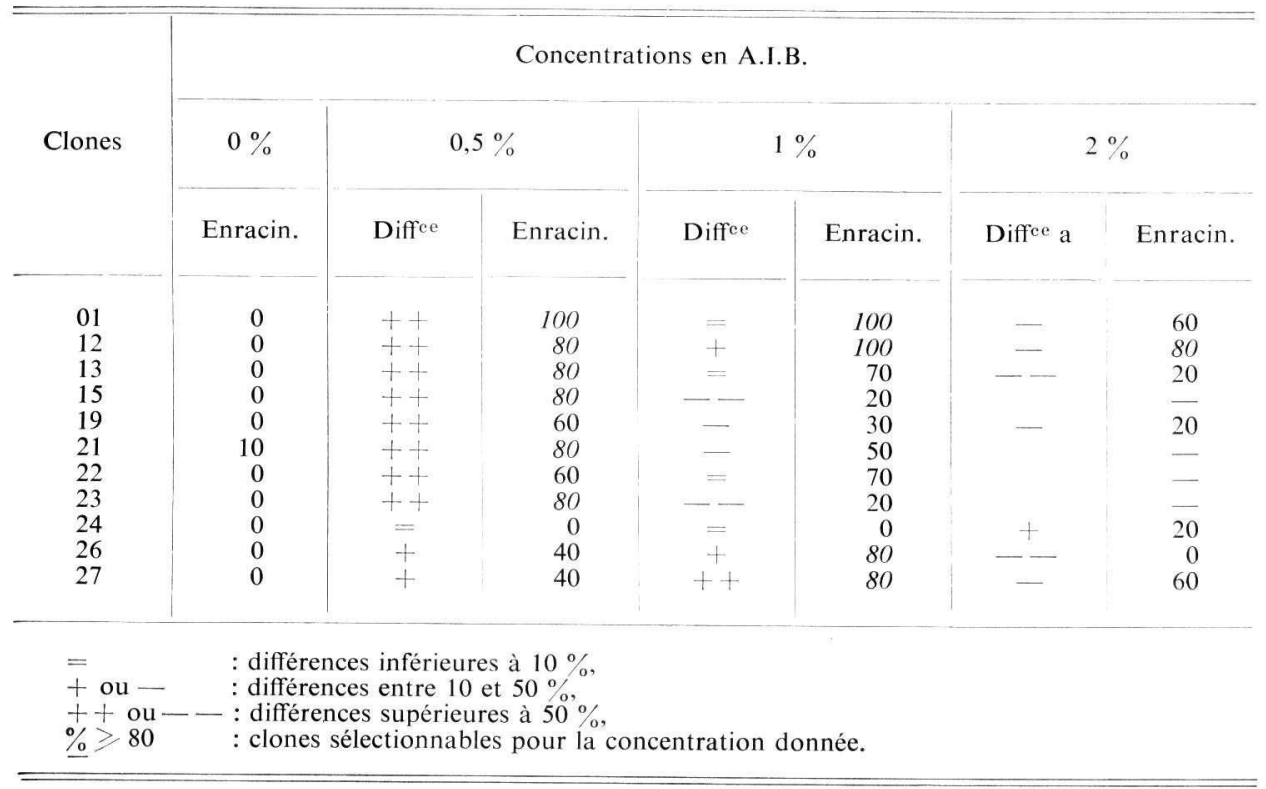

Bien qu'il soit difficile de s'appuyer sur ces résultats pour tirer des conclusions définitives, et en gardant à l'esprit les restrictions précédentes, il semble qu'il faille remettre en question notre base pratique de sélection sur une seule concentration auxinique à $1 \%$ d'A.I.B. En effet, comme nous l'avons déjà dit, $0,5 \%$ d'A.I.B. serait préférable. De même, si nous conser- 
vons les clones dont la réussite est supérieure à $80 \%, 6$ sont sélectionnables avec $0,5 \%$ d'A.I.B. et 4 avec $1 \%$. Mais seuls 2 clones ( 01 et 12 ) sur les 8 donnent des résultats valables pour les deux concentrations étudiées. Nous avons donc une perte de $25 \%$ ou $50 \%$ du matériel dans cet exemple, suivant que l'on choisit comme base de test des concentrations de 0,5 ou $1 \%$ d'A.I.B.

\section{III. - CONCLUSION}

Les résultats que nous venons de présenter bien que parcellaires permettent de tirer un certain nombre de conclusions provisoires qui demandent confirmation et précision.

Si la multiplication végétative du Douglas semble aisée à l'état jeune, elle demande certaines conditions. Un milieu très filtrant est indispensable dans le cas d'utilisation d'arrosage par nébulisation fréquente. Un traitement auxinique est nécessaire : une concentration de poudre à $0,5 \%$ d'A.I.B. donnant les meilleurs résultats. Dans nos conditions expérimentales, le gain apporté par le traitement auxinique s'accompagne d'une augmentation très nette de la mortalité.

Ce sont les dernières ramifications des rameaux latéraux qui donnent les meilleurs résultats. Nous pouvons, de ce fait, bénéficier de 20 à 40 boutures standards pour un sujet au niveau de la troisième couronne, quantité minimum pour procéder à des tests valables.

Nous avons pu déceler une très grande variabilité individuelle de l'aptitude du Douglas au bouturage.

Dans un ensemble de sujets, un certain nombre de clones se révèlent « supérieurs ». Au point de vue sélection pour l'aptitude au bouturage, ces clones « sélectionnables 》 représentent en moyenne 20 à $25 \%$ de la population initiale. Compte-tenu des rejets qui devront être effectués plus tard au niveau clonal (performances adultes du sujet tête de clone (densité, homogénéité du bois, production...) ainsi que développement général des boutures), un grand nombre de sujets doit être sélectionné et testé, afin d'obtenir un minimum de 50 clones utilisables pour la constitution de variétés multiclonales. Par ailleurs, l'existence de corrélations négatives entre certains caractères économiques et l'aptitude au bouturage, peut venir perturber le schéma et l'intensité finale de la sélection.

Du fait des nombreux biais qui peuvent agir aussi bien avant que pendant le test, l'aptitude d'un clone à l'enracinement ne doit pas être jugée sur un seul test. Trois essais devraient donner une réponse satisfaisante.

Des expérimentations complémentaires sont nécessaires afin de définir si la sélection doit s'opérer à partir d'une ou plusieurs concentrations auxiniques. L'utilisation par exemple de deux populations de clones bouturables à partir de 2 traitements auxiniques différents peut être envisageable si l'on augmente de façon notable le nombre de clones utilisables.

Ces études devront être poursuivies en multipliant le nombre de clones testés. Elles seront complétées par l'étude du développement ultérieur des boutures enracinées obtenues. Une attention particulière sera portée à la structure du système racinaire, au rétablissement rapide et au maintien de l'orthotropisme ainsi qu'à la croissance. Elles devront aussi tenir compte des facteurs économiquement envisageables en fonction du but recherché : plants pour vergers à graines ou plants de variétés multiclonales pour reboisement. 


\section{SUMMARY \\ PRELIMINARY STUDIES ON THE SELECTION OF CLONES FOR THEIR ABILITY TO BE PROPAGATED BY CUTTINGS \\ (Pseudotsuga menziesii FRANCO).}

The purpose of this work is to study the possibility of using cuttings for the creation of clone seed-orchards and multiclonal varieties. Three experiments have been relaised in 1970 and 1972 with selected clones from provenance tests (Epinal, planted in 1965) and from stands (Beaujolais, mean age $60 \mathrm{y}$.$) .$

The cutting is a 15 centimeters long ( 6 inches) part of the current year shoot. It is cut of the mother tree in February and March. Before being dipped in I B A mixed with talcum powder at a 0,$5 ; 1$ or $2 \%$ ratio, the basal part $(3$ to $4 \mathrm{~cm})$ of the cutting has its needles taken of. The rooting is realised in a polythene greenhouse with a make and break mist. The results are noted 6 to 8 months later.

The cuttings are very sensitive to a too high moisture of the sand medium. Although it has a significantly rooting action, the auxin increases mortality.

The best results have been obtained with lateral cuttings dipped in $0,5 \% \mathrm{I} \mathrm{B} \mathrm{A} /$ talcum powder mixture.

A satisfactory similarity correlation for rooting has been found for 14 clones tested in 1970 and 1972. Three clones gave discordant results.

The average rooting percentages are as follow :

$1970: 41 \%$ for 27 clones of Epinal provenance test.

$1972: 63,5 \%$ for 14 of these clones (the value for 1970 was only $46,5 \%$ for this sample).

$1972: 18 \%$ for 49 clones selected in plantations.

This shows that older trees have a much lower rooting ability than younger ones.

The between clone variability is very high. There also appears a clone - I B A concentration interaction. Rooting capacity reaching $80 \%$ have been found for differents I B A levels :

$0,5,1$ and $2 \%$ for 1 clone

0,5 and $1 \%$ for 1 clone

$1 \%$ for 2 clones

$0,5 \%$ for 4 clones.

For a given treatment, about $20 \%$ of the clones of the studied populations have a high rooting ability and can be selected for this characteristic. The results of these tests will have to be checked for several years. Different auxin concentrations will have to be used. This will unable the selection of a good quantity of clones for their ability of being propagated by cuttings.

\section{ZUSAMMENFASSUNG}

VORLÄUFIGE VERSUCHE ZUR AUSWAHL VON ZUR STECKLINGSVERMEHRUNG GEEIGNETEN KLONEN DER DOUGLASIE

(Pseudotsuga menziesii fRANCO).

Das Ziel dieses Versuches ist die Verwendung von Stecklingen entweder zur Anlage von Samenplantagen mit Klonen oder zur Züchtung multiklonaler Varietäten. Drei Versuche wurden von 1970 bis 1972 mit Material von ausgewählten Klonen aus Vergleichspflanzungen von Herkünften (Epinal, Pflanzung aus dem Jahre 1965) und aus Beständen (Beaujolais, mittleres Alter 60 Jahre) durchgeführt.

Die Stecklinge (15 cm lange Teile von Jahrestrieben) wurden im Februar und März eingesammelt. Vor der Behandlung mit einer Mischung von A.I.B. und Talk $(0,5 \%, 1 \%$ oder $2 \%$ A.I.B.) wurden die Nadeln an der Basis auf 3 bis $4 \mathrm{~cm}$ Länge entfernt. Die Stecklinge werden in einem 
Glashaus (Plastik) unter intermittierendem « Mist» herangezogen. Die Messungen erfolgten 6 bis 8 Monate nach der Einbringung.

Die Stecklinge reagieren sehr stark auf einen Feuchteüberschuss im Sandsubstrat. Die Auxinbehandlung erhöht signifikant die Bewurzelung jedoch bringt sie auch eine deutliche Erhöhung der Mortalität mit sich.

Die besten Ergebnisse wurden mit Seitentrieben 2. Ord. und Behandlung mit einer Talkmischung von $0,5 \%$ A.I.B. erzielt.

Bei 14 Klonen wurde eine gute Übereinstimmung der Bewurzelung zwischen den Versuchen 1970 und 1972 beobachtet. Nur 3 Klone ergaben stark abweichende Ergebnisse.

Folgende mittlere Bewurzelungsprozente wurden erzielt :

1970 : $41 \%$ bei 27 Klonen aus Epinal;

1972 : $63,5 \%$ bei 14 Klonen, welche innerhalb der obgenannten 27 Klone ausgewählt wurden $(46,5 \%$ im Jahre 1970);

1972 : $18 \%$ bei 49 Klonen aus Beständen im Beaujolais.

Die von älteren Bäumen stammenden Stecklinge scheinen ein geringeres Bewurzelungsvermögen zu haben.

In allen Versuchen konnte eine sehr starke Variabilität zwischen Klonen bei gleicher Behandlung festgestellt werden. Desgleichen zeigt sich eine gewisse Variabilität bei verschiedenen Auxinkonzentrationen. Bei 11 Klonen konnte ein maximales Bewurzelungsprozent mit folgenden A.I.B. - Konzentrationen erzielt werden :

- $0,5 \%, 1 \%$ und $2 \%: 1$ Klon

$-0,5 \%$ und $1 \%: 1$ Klon

$-1 \%: 2$ Klone

$-0,5 \%: 4$ Klone

Von den untersuchten Populationen bei gegebener Behandlung, können ca. $20 \%$ als für die Stecklingsvermehrung geeignet ausgewählt werden. Die erhaltenen Ergebnisse lassen eine Fortsetzung der Versuche während mehrerer Jahre und allenfalls mit weiteren Auxinkonzentrationen erfolgversprechend erscheinen um mit Sicherheit ein Maximum an zur Stecklingsvermehrung geeigneten Klonen auswählen zu können.

\section{RÉFÉRENCES BIBLIOGRAPHIQUES}

Bouvarel P. et Turpin P. — Communication personnelle d'expériences effectuées en 1954 et 1955 . (Non publié).

Brix H. and BARker H., 1969. - Rooting of Douglas-fir and western hemlock cuttings. Dep. Fish. Forest Can. Bi-mon. Res. Note 25 (3), 22.

Brix H. and BARKer H., 1971. - Trials in Rooting of Douglas-fir cuttings by a paired-cutting technique. Can. J. Forest Res. 1 (2), 121-125.

CoRnu D., 1971. - Rapport sur les travaux effectués au Québec. Coopération technique 1970-1971. (Non publié).

Heaman J.-C. and Owens J.-N., 1972. - Callus formation and root initiation in stem cuttings of Douglasfir (Pseudotsuga menziesii (Mirb.) Franco). Can. J. Forest Res., 2 (2), 121-134.

Heinrich J.-C., 1969. — Le bouturage du Douglas. Compte-rendu d'activité 1968 de 1'A.F.O.C.E.L., 4-33.

Roberts A.-N., 1969. - Timing in cutting propagation as related to developmental physiology. Proc. Pl. Propag. Soc., 19, 77-82. 